\title{
Initial Model Catalogue for Galaxy Evolution
}

\author{
Naohito Nakasato $^{1,2}$ \\ ${ }^{1}$ Department of Astronomy, University of Tokyo, Hongo 7-3-1, Bunkyo-ku Tokyo, 113-0033, Japan \\ ${ }^{2}$ E-mail: nakasato@ astron.s.u-tokyo.ac.jp
}

Received 2003 October 1, accepted 2004 January 5

\begin{abstract}
We have computed full hydrochemodynamical evolution for 150 initial models of protogalaxies with our chemodynamical SPH code named GENSO. Various parameters for all models are identical except for a seed for a random number generator. In other words, all models have similar global properties but have the different merging history that leads to a different evolution in each model. Results of the series of computations have two main applications. Firstly, we have an initial model catalogue for subsequent modelling of galaxy evolution. Since the resulting evolution depends strongly on the initial phase of the particle distribution, it is crucial to find a suitable initial model when we model a specific real galaxy in the Universe, notably the Milky Way in our case. We will make a precise chemical and dynamical model of the Milky Way out of 150 models in our initial model catalogue. Secondly, we can obtain a large variety of global histories of physical values such as star formation, metallicity in the ISM and stellar components, and Type II and Ia supernova rates. For example, the resulting total star formation history shows the peak at a high redshift $z \sim 6$ and the peak value is $\sim 280 \mathrm{M}_{\odot} \mathrm{yr}^{-1} \mathrm{Mpc}^{-3}$. Also, the Type Ia rate obtained has a peak at $z \sim 3.5$. All of our results and model catalogue are publicly available from our website for those who wish to model galaxy evolution.
\end{abstract}

Keywords: ISM: clouds — Galaxy: kinematics and dynamics — methods: $N$-body simulations

\section{Introduction}

In the current, most plausible, cold dark matter (CDM) cosmology, the evolution of galaxies involves the progressive merging of sub-galactic clumps (e.g. Baugh et al. 1996). Due to these progressive merger events, galaxies have formed and evolved. Such merger events could trigger starbursts depending on the mass of each merging object. In other words, star formation history reflects the strength of the interaction between a galaxy and merging objects. Also, several merger events strongly affect the development of the morphology of galaxies as assumed in semi-analytic models. In the most advanced semi-analytic models (e.g. Kauffmann et al. 1999), $N$ body simulations of dark matter particles are used to obtain the merging history of halos. By combining the description of radiative cooling, hydrodynamics, and star formation with the obtained merging history, such models have successfully explained various observational constraints. Here, we show the results of full numerical galaxy evolution models. In contrast to the semi-analytic models, we use our high resolution smoothed particle hydrodynamics (SPH) models to compute the evolution of small regions of the early Universe. We have performed over 100 high-resolution SPH simulations and analysed the properties of such models. Our results clearly show that the dynamical evolution of merger events is a most effective process to determine star formation history and galaxy morphologies. There are two main purposes of this work. First, detailed analysis of the models enables us to obtain insights into galaxy evolution. Another intention is to use the results of the present work as an initial model catalogue for subsequent modelling of galaxy evolution. Since the simulation results depend strongly on the merging history, it is crucial to find or select a suitable initial model when we model a specific real galaxy in the Universe, notably the Milky Way in our case. Out of over 100 models, we will find a reasonable initial model that can be used to construct a precise dynamical and chemical model for the Milky Way.

\section{Method and Initial Models}

To model galaxy evolution, we used our chemodynamical SPH code. Since details of our code have been reported in Nakasato \& Nomoto (2003, hereafter NN2003), we only mention here the main features of our SPH code: (a) gravity interaction is computed with GRAPE (Sugimoto et al. 1990); (b) the SPH formulation is the same as in Navarro \& White (1993); (c) the smoothing length of the SPH method is controlled with the method by Thacker et al. (2000); (d) the metallicity-dependent radiative cooling function with MAPPINGS III is in NN2003; and (e) star formation, stellar feedback (including Type II and Ia supernovae), and chemical enrichment algorithms are also described in NN2003. We call our chemodynamical SPH code for galaxy evolution GENSO (Galaxy EvolutioN with the Sph methOd).

For our purpose of simulating several dozen galaxy models, we need to choose an efficient method to set up 
initial models. A standard method to set up a cosmological initial model for galaxy evolution is the so-called resimulation technique (Navarro \& White 1994). In this technique, one selects a halo with the desired properties from large scale cosmological $N$-body simulations. In contrast, we adopt the path integral method that directly generates a desired halo (Bertschinger 1987). With this method, we can efficiently generate any number of halos with similar properties, i.e. the mass and size in the present case. This efficiency of generating desired halos is an advantageous point over the resimulation technique. On the other hand, with our method, we have to use the isolated boundary condition. Consequently, we neglect the cosmological tidal field and possibilities of late infall of external material in the present work. Due to the neglect of the cosmological tidal field, we artificially add angular momentum to the initial velocity field of a model.

What we will try to do using the results of this study is to understand the evolution of a Milky Way-sized halo. For this purpose, we generate a number of $3 \sigma$ overdense spherical regions with mass $\sim 10^{11}-10^{12} \mathrm{M}_{\odot}$. Each model has similar mass but is expected to have a different merging history that leads to a different star formation history. Details of how to set up an initial model are described in NN2003, except that we adopt $\Lambda$ CDM-dominated cosmology in the present work. Here, we summarise parameters for our initial models: (a) $\Lambda \mathrm{CDM}$ cosmology with $\Omega_{\text {matter }}=0.3$ and $\Omega_{\Lambda}=0.7$ is assumed; (b) we set the Hubble constant $H_{0}=65 \mathrm{~km} \mathrm{~s}^{-1} \mathrm{Mpc}^{-1}$; (c) the CDM spectrum with $\sigma_{8}$ at $z=0$ equals 1.0 ; (d) the starting redshift is $\sim 24$; (e) the baryon fraction is $10 \%$; (f) the model region is a $3 \sigma$ spherical region; $(\mathrm{g})$ the radius of the sphere is $\sim 1.5 \mathrm{Mpc}$ (comoving units); (h) the Plummer softening lengths are $\epsilon_{\mathrm{SPH}}=\epsilon_{\mathrm{star}}=0.5 \mathrm{kpc}$, and $\epsilon_{\text {dark }}=1.0 \mathrm{kpc}$; and (i) the mean number of neighbouring particles for the SPH method is $\sim 60$. The parameters related to star formation and chemical evolution are the same as used in NN2003. As noted already, each simulated region in the present work is spherical and isolated. Thus, initially, we make a sphere in rigid rotation with angular velocity $5 \times 10^{-17} \mathrm{~s}^{-1}$ (the same value used in NN2003), and a resulting spin parameter for each model is in the range $0.11-0.15$. Since the initial phase of the particle distribution for each model is different, the mass of the sphere is not constant and is distributed in the range $(5-7) \times 10^{11} \mathrm{M}_{\odot}$. In all models, the initial number of particles is $\sim 50000$ in total (an exact number of particles is different for each model with the same reason above), where half are the dark matter particles and the others the gas particles. We have computed the full chemical and dynamical evolution up to $z \sim 1.28$ ( 5 Gyr) for these initial models, with radiative cooling, star formation, and stellar feedback always on. In total, we have computed 150 full chemodynamical models. In the present paper, we only discuss global trends in our results. Detailed analysis of specific models and further precise modelling of the Milky Way will be presented elsewhere.

\section{Results}

Since we add a relatively large angular velocity to the initial velocity field of the models, we expect that a fair fraction of the models result in a disk galaxy. To see this trend, we first fit the surface stellar density profile of a model at $z \sim 1.28$ with the summation of exponential and de Vaucouleurs's profiles as

$$
\begin{aligned}
\Sigma(R)= & \Sigma_{\text {disk }} \exp \left(-R / R_{\text {disk }}\right) \\
& +\Sigma_{\text {bulge }} \exp \left\{-7.67\left[\left(R / R_{\text {bulge }}\right)^{0.25}-1\right]\right\},
\end{aligned}
$$

where $R$ is the distance from the galactic centre. In each model, we take the centre of gravity of the stellar particles as the centre of the coordinates and align the angular momentum vector with the z-axis. Out of 150 models, 99 models are well-fitted with this profile, where we make constraints on $R_{\text {disk }}$ and $R_{\text {bulge }}$ as $1.0 \leq R_{\text {disk }} \leq 5.0 \mathrm{kpc}$ and $0.1 \leq R_{\text {bulge }} \leq 2.0 \mathrm{kpc}$. The relation between $\Sigma_{\text {disk }}$ and $R_{\text {disk }}$ for these well-fitted models is shown in Figure 1. Note that this figure shows the relation at $z \sim 1.28$ and subsequent evolution will certainly change the parameters when we could evolve the models further. It seems there is a weak correlation between $\Sigma_{\text {disk }}$ and $R_{\text {disk }}$. As fiducial parameters for selecting candidates for subsequent modelling of the Milky Way we make the constraint $3.0 \leq R_{\text {disk }} \leq 4.0 \mathrm{kpc}$ and obtain 48 candidates. Figure 2 shows the relation between $\Sigma_{\text {bulge }}$ and $R_{\text {bulge. }}$ In the case of the bulge parameters, there is a tight relation between these two parameters. In either case, similar correlations between the surface brightness and the scale length for bulge and disk components are observed (e.g., Kent 1985; Binney \& Merrifield 1998).

A number (18) of the other 51 models failed to fit because there is still no dominant galaxy, due to the longer evolution time scale than in the well-fitted disk models. This difference is shown in Figure 3. These panels show the edge-on view for stellar particles: (a) the most prominent disk model (left-hand panel); and (b) a failed model (right-hand panel). The models like the former one tend to collapse earlier than the models like the latter. In such

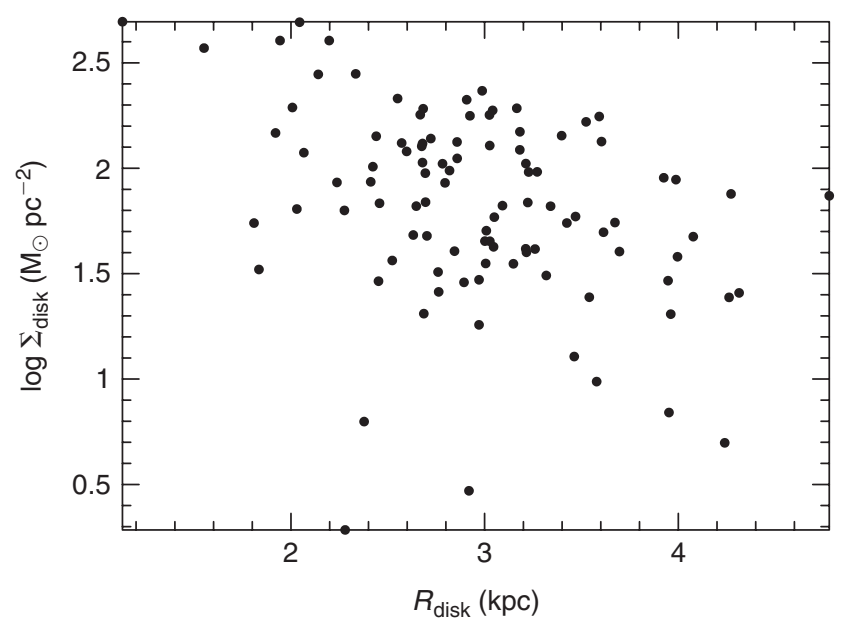

Figure 1 The relation between disk parameters $\Sigma_{\text {disk }}$ and $R_{\text {disk }}$. 
prominent disk models, the star formation history is quite similar to that of the model presented in NN2003: (a) early star formation begins at $z \sim 15-17$ and increases rapidly due to global gravitational contraction; (b) star formation peaks $\left(\sim 60 \mathrm{M}_{\odot} \mathrm{yr}^{-1}\right)$ at $z \sim 4-6$ and a large fraction of the bulge component forms at this stage; and (c) subsequent star formation occurs mainly in disk regions and produces a disk component. On the other hand, star formation for the model shown in the right-hand panel of Figure 3 does not show a strong peak but a weak and wider peak at $z \sim 3$ with the maximum value of $\sim 20 \mathrm{M}_{\odot} \mathrm{yr}^{-1}$.

Each model shows a quite different star formation history as expected. Here, we sum the star formation rate for all models and present it in Figure 4. To normalise

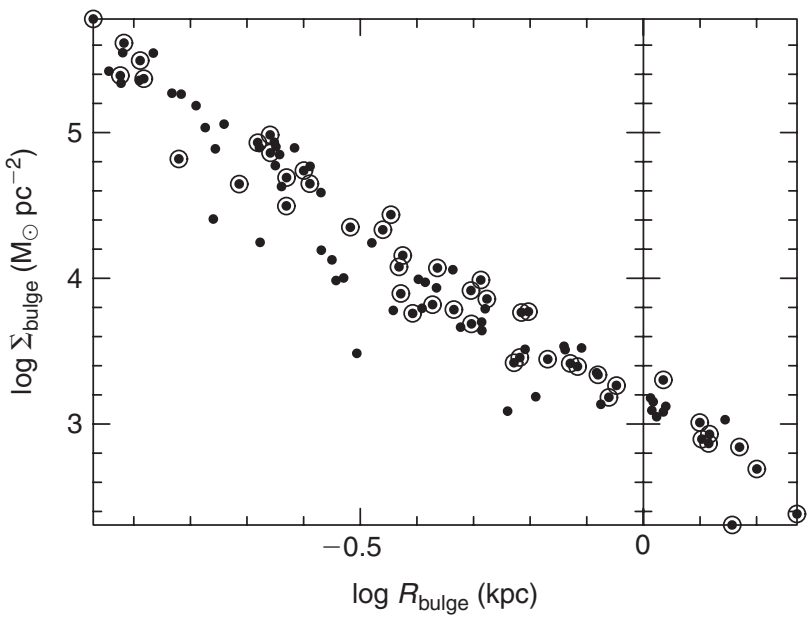

Figure 2 The relation between bulge parameters $\Sigma_{\text {bulge }}$ and $R_{\text {bulge }}$. The dotted circles correspond to 48 candidate models with $3.0 \leq R_{\text {disk }} \leq 4.0 \mathrm{kpc}$ (see text for details). the star formation rate, we assume that the volume for normalisation in each model is always spherical with the adopted comoving radius $(\sim 1.5 \mathrm{Mpc})$. In Figure 4 , we plot the total star formation history with a solid line. The lower dotted line shows the contribution from the 99 disk galaxy models explained above. The peak of star formation rate occurs at $z \sim 6$ in either case. This merely reflects that the typical collapse redshift (which depends on the initial density amplitude and the initial spin parameter of the sphere), when strong merging events occur at the centre as reported in NN2003, is $z \sim 6$. The contribution from the disk galaxy models decreases at the late stage $z<6$. This means that such disk models evolve faster than other models, as already noted.

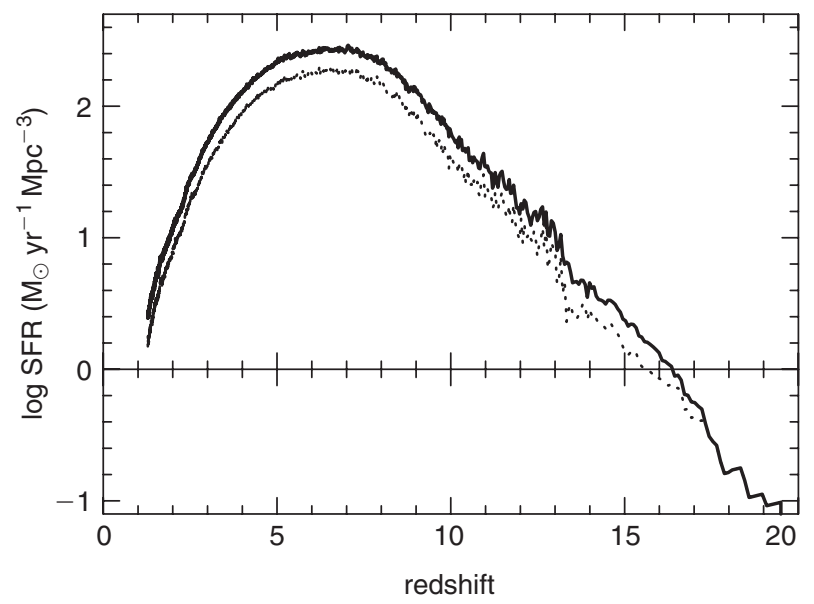

Figure 4 The upper solid line shows the model averaged star formation history by summing the star formation rate for 150 models. The lower dotted line shows the history for 99 well-fitted disk models.
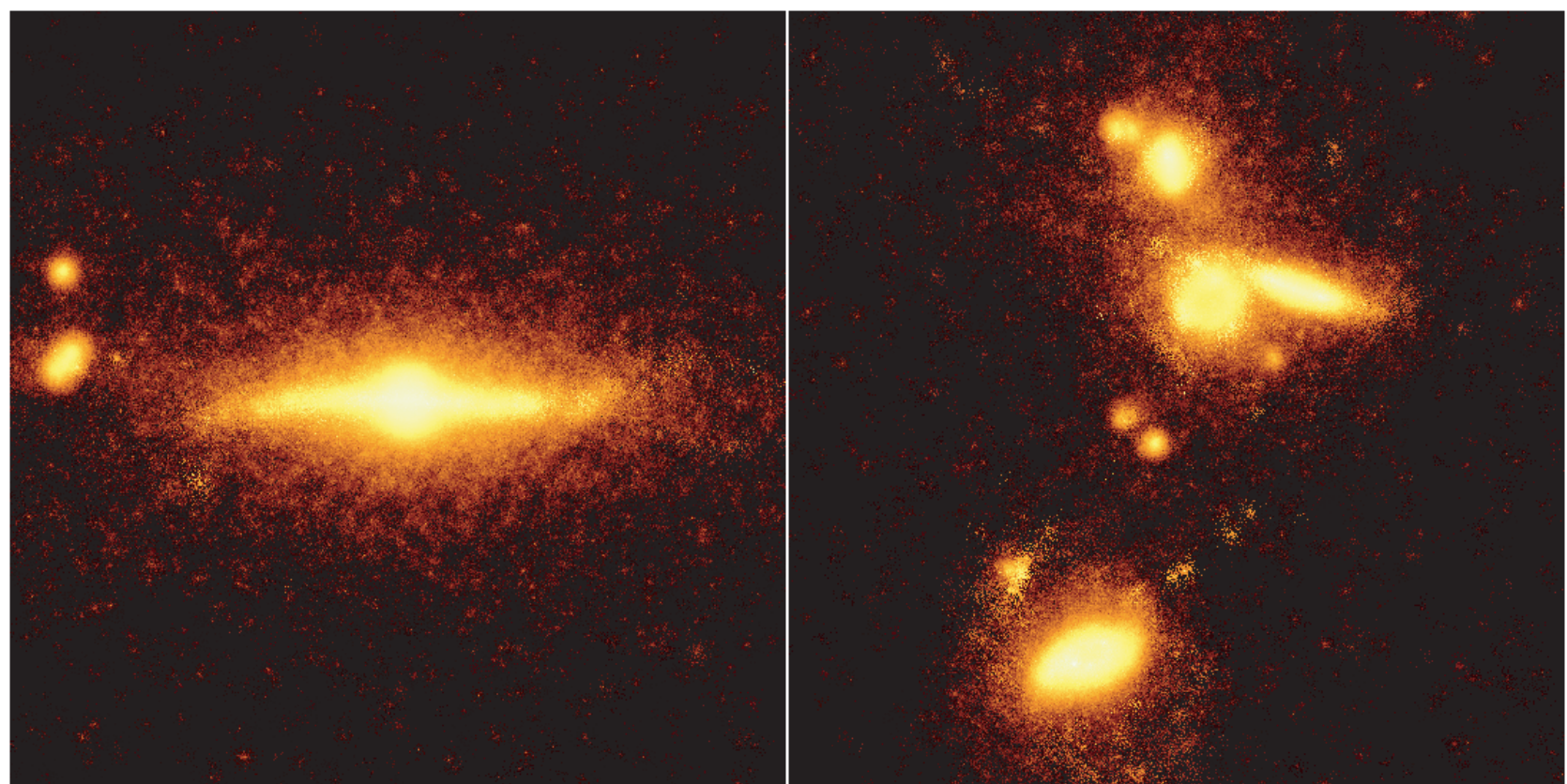

Figure 3 Example of morphology obtained for different initial models showing the edge-on view for stellar particles at $z \sim 1.28$; the scale of the panels is $30 \mathrm{kpc}$. (a) The most prominent disk model (left-hand panel), and (b) a failed model (right-hand panel). In the latter model, the overall evolution is slower than in the former model. As a result, small sub-galactic clumps (dwarf galaxies) are still evolving at this stage. 


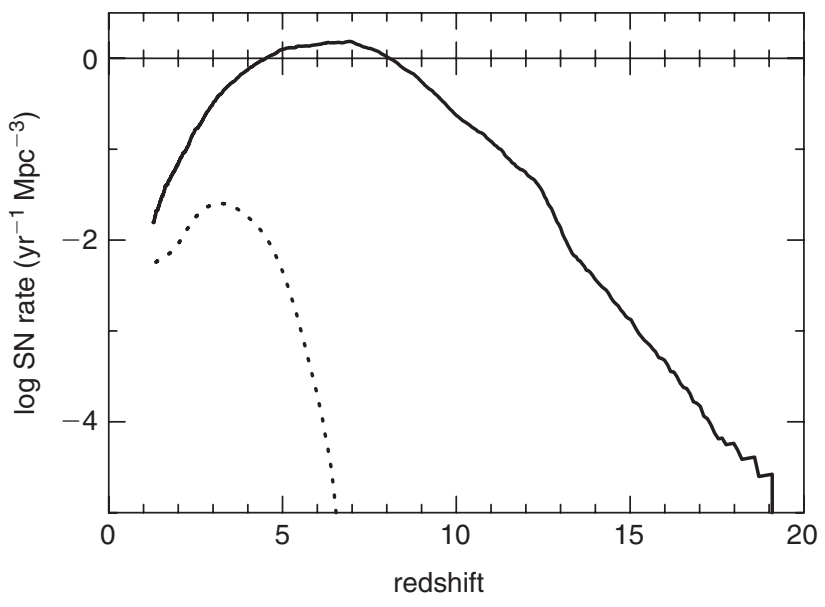

Figure 5 The upper solid line shows the Type II supernova rate obtained by our model. The Type Ia supernova rate is shown by the dotted line.

Similarly, Figure 5 shows the supernova rate for Type II and Ia supernovae in the solid and dotted lines, respectively. The method of normalisation is the same as the above method. Naturally, the Type II rate peaks at the same redshift as the global star formation history presented in Figure 4. With our adopted Type Ia supernova model (Kobayashi et al. 1998), the peak of the Type Ia supernova rate occurs at $z \sim 3.5$. Note that the delay time, which is the time difference between the birth time of a single stellar population and the occurrence of the first Type Ia supernova explosion, is $\sim 0.5 \mathrm{Gyr}$. Our result is an updated version of Kobayashi et al. (2000), which predicted the cosmic supernova rates history based on one-zone chemical evolution models. Their prediction for the cosmic Type Ia supernova rate was that the peak occurs at $z \sim 2.5$ for a cluster environment with cosmological parameters of $\Omega_{\text {matter }}=0.2, \Omega_{\Lambda}=0.0$, and $H_{0}=50 \mathrm{~km} \mathrm{~s}^{-1} \mathrm{Mpc}^{-1}$.

There are several factors that alter our predictions of global histories of star formation and supernova rates. One is that our predictions for star formation and supernova rate are clearly overestimated for a given comoving volume. This is because we only consider high density peaks in the early Universe. On the other hand, lower density peaks collapse at a later stage of the history of the Universe and should not have strong influences on the prediction at the earlier Universe. Accordingly, we believe that the shape for the global history of our results is reasonable and we should correct the normalisation by accounting for the effect of neglected regions when we make a detailed comparison between our results and other predictions. Another point, which is a more severe factor in the present case, is that the adopted description of stellar feedback (i.e. the energy deposited by stellar activities is distributed as thermal energy for simplicity) in our model is too effective to solve the so-called 'overcooling' problem. As a result, the early star formation rate, especially, may be overestimated. There are already a number of attempts (e.g. Thacker \& Couchman 2001; Sommer-Larsen et al. 2003) to solve this problem and we will need a more sophisticated description for stellar feedback processes in future work.

\section{Conclusion}

In this paper, we present the results of hydrochemodynamical simulations for 150 initial models. Each model is a high density $(3 \sigma)$ spherical region with a CDM spectrum. The mass of the models is $\sim(5-7) \times 10^{11} \mathrm{M}_{\odot}$ depending on the initial phase of the particle positions in each model. The mass and size are selected to match a similar scale to the Milky Way since one of our intentions is to make an initial model catalogue for a subsequent modelling of the Milky Way. The different initial phase results in a large variety of star formation histories in each model. In other words, the resulting distribution of stellar particles (i.e. morphology of galaxies) and scale parameters (e.g. disk scale length) depend strongly on the merging history yielded by the initial phase. We have evolved 150 models from $z \sim 24$ to $z \sim 1.3$. By fitting the stellar surface density at $z \sim 1.3$, we find that 99 out of 150 models result in a disk galaxy. The bulge component of these disk galaxy models shows a clear relation between the central density and the core radius. The total star formation history obtained peaks at $z \sim 6$ with a rather high value of $\sim 280 \mathrm{M}_{\odot} \mathrm{yr}^{-1} \mathrm{Mpc}^{-3}$. Also, the Type Ia supernova rate obtained has a peak at $z \sim 3.5$. In future work, we will make a precise chemical and dynamical model of the Milky Way by selecting an appropriate initial model out of 150 models. Our results and initial model catalogue are publicly available at our website, http://www.astron.s.utokyo.ac.jp/ nakasato/CD/, for those who wish to model galaxy evolution.

\section{Acknowledgments}

Most of the computation has been performed with the GRAPE cluster at the Astronomical Data Analysis Center of the National Astronomical Observatory, Japan. A part of this work has been supported by JSPS Research Fellowships for Young Scientists (05127).

\section{References}

Baugh, C. M., Cole, S., \& Frenk, C. S. 1996, MNRAS, 283, 1361 Bertschinger, E. 1987, ApJ, 323, L103

Binney, J., \& Merrifield, M. 1998, Galactic Astronomy (Princeton: Princeton University Press)

Kauffmann, G., Colberg, J. M., Diaferio, A., \& White, S. D. M. 1999, MNRAS, 303, 188

Kent, S. 1985, ApJS, 59, 115

Kobayashi, C., Tsujimoto, T., \& Nomoto, K. 2000, ApJ, 539, 26

Kobayashi, C., Tsujimoto, T., Nomoto, K., Hachisu, I., \& Kato, M. 1998, ApJ, 503, L155

Nakasato, N., \& Nomoto, K. 2003, ApJ, 588, 842

Navarro, J. F., \& White, S. D. M. 1993, MNRAS, 265, 271

Navarro, J. F., \& White, S. D. M. 1994, MNRAS, 267, 401

Thacker, R. J., \& Couchman, H. M. P. 2001, ApJ, 555, L17

Thacker, R. J., Tittley, E. R., Pearce, F. R., Couchman, H. M. P., \& Thomas, P. A. 2000, MNRAS, 319, 61

Sommer-Larsen, J., Götz, M., \& Portinari, L. 2003, ApJ, 596, 47

Sugimoto, D., Chikada, Y., Makino, J., Ito, T., Ebisuzaki, T., \& Umemura, M. 1990, Nature, 345, 33 